\title{
Vacuum entanglement governs the bosonic character of magnons
}

\author{
Tomoyuki Morimae* \\ Laboratoire Paul Painlevé, Université Lille 1, F-59655 Villeneuve d'Ascq Cedex, France
}

(Dated: December 27, 2018)

\begin{abstract}
It is well known that magnons, elementary excitations in a magnetic material, behave as bosons when their density is low. We study how the bosonic character of magnons is governed by the amount of a multipartite entanglement in the vacuum state on which magnons are excited. We show that if the multipartite entanglement is strong, magnons cease to be bosons. We also consider some examples, such as ground states of the Heisenberg ferromagnet and the transverse Ising model, the condensation of magnons, the one-way quantum computer, and Kitaev's toric code. Our result provides insights into the quantum statistics of elementary excitations in these models, and into the reason why a non-local transformation, such as the Jordan-Wigner transformation, is necessary for some many-body systems.
\end{abstract}

PACS numbers: 03.65.Ud, 03.67.Bg, 03.67.Mn

\section{INTRODUCTION}

The study of condensed matter physics from the view point of quantum information science [1] is one of the most active research topics in today's quantum manybody physics. Plenty of important results have been obtained and they have contributed to the recent mutual fertilization between those two fields [2-4]. Among many characteristics of quantum many-body systems, ground states and low-energy excited states are fundamental targets in condensed matter physics [5]. Indeed, a great deal of research has been performed on the behavior of an entanglement in ground states [6], and these researches have revealed several novel aspects of quantum ground states including the entanglement divergence at a quantum critical point [7] and the role of an entanglement in efficient approximations of many-body ground states [8].

Although ground states of many-body Hamiltonians are often very complicated, it is notorious that excited states are more complicated due to their high degeneracy. Because of this complexity of excited states, the study of excited states from the view point of quantum information is very slow and not yet fully developed. This seems to cause the severe lack of knowledge about the implementation of quantum computation on low-temperature solids. In the long history of condensed matter physics, one solution to cope with such complicated excited states has been the idea of "elementary excitations" [9]. A lowenergy excited state of a many-body Hamiltonian can be interpreted as a ripple of the degrees of freedom under consideration (such as the electromagnetic field or the density of atoms), and this ripple is second quantized to have (quasi-)particles (such as photons or phonons) whose lifetimes are not necessarily infinite. Such particles are called "elementary excitations" [9]. For a magnetic material, such as a ferromagnet or an antiferromagnet, such a quantum of a spin wave is called "magnon".

*Electronic address: morimae@gmail.com
Low-energy excited states of a magnetic Hamiltonian are labelled with the density and wavenumbers of magnons excited on the vacuum, and this labelling enables a systematic study of otherwise highly complicated low-energy excited states. Indeed, this "magnon picture" has been applied to several researches in quantum information, such as entanglement properties of spin systems [10, 11] and the transfer of quantum information through a spin chain [12]. Considering facts that elementary excitations are at the heart of quantum many-body physics, and that a quantum information processing will be ultimately implemented on a quantum many-body system, we cannot overestimate the role of elementary excitations played in quantum information science.

Recently, Law [13] and Chudzicki, Oke, and Wootters [14] have shown an interesting relation between the bosonic character of composite fermions and the entanglement between these fermions. Their main result is that if the entanglement is strong, an indicator of the bosonic character of the composite fermions approaches its ideal bosonic value. In other words, the composite fermions behave as bosons, when the entanglement is strong. Their researches have opened the door to the study of the quantum statistics from the view point of entanglement.

In this Rapid Communication, we study how the bosonic character of magnons is governed by the amount of a multipartite entanglement in the vacuum state on which magnons are excited. Our main result is Eq. (11), which shows that if the multipartite entanglement is strong, magnons cease to be bosons.

One might claim that our result "strong entanglement means weak bosonic character" is contradictory to the result "strong entanglement means strong bosonic character" by Law [13], Chudzicki, Oke, and Wootters [14]. However, note that the entanglement we consider here is different from that considered by them: we see a multipartite entanglement in the vacuum on which particles are excited, whereas they see the bipartite entanglement between two excited particles. Of course, it would be interesting to explore a relation between our result and 
theirs. It is, however, beyond the scope of the present paper, and therefore left for a future study.

As examples, we consider several typical quantum many-body systems, such as the Heisenberg ferromagnet, the transverse Ising model, the one-way quantum computer [15], and Kitaev's toric code [16]. We will see that our result Eq. (11) provides an insight into the quantum statistics of elementary excitations in these models. We will also see that our result explains why a non-local transformation, such as the Jordan-Wigner transformation [17], is necessary for some many-body systems, such as the transverse Ising model. Furthermore, we also consider the condensation of magnons. Since a state where a macroscopic number of magnons are excited is highly entangled [10], Eq. (1) also explains the often-pointed-out fact that magnons cease to be bosons when their density is high.

We would like to emphasize that the result of this Rapid Communication sublimates plenty of recent calculations of the multipartite entanglement in ground states from mere academic studies into meaningful applications in condensed matter physics: calculations of the multipartite entanglement in ground states are useful to obtain an insight into the quantum statistics of elementary excitations excited there.

\section{MAGNON}

For simplicity, let us consider the one-dimensional lattice of $N$ spin-1/2 particles. The creation operator $\hat{M}_{k}^{\dagger}$ of the magnon with wavenumber $k$ is defined by

$$
\hat{M}_{k}^{\dagger} \equiv \frac{1}{\sqrt{N}} \sum_{l=1}^{N} e^{i k l} \hat{\sigma}_{l}^{+}
$$

where $\hat{\sigma}_{l}^{+}$is the "spin-ladder" operator on site $l$ defined by $\hat{\sigma}_{l}^{+}|0\rangle_{l}=|1\rangle_{l}$ and $\hat{\sigma}_{l}^{+}|1\rangle_{l}=0$. Here, $|1\rangle_{l}$ and $|0\rangle_{l}$ are eigenvectors of Pauli's $z$ operator $\hat{\sigma}_{l}^{z}$ on site $l$ corresponding to eigenvalues \pm 1 , respectively. The annihilation operator of the magnon with wavenumber $k$ is the Hermitian conjugate $\hat{M}_{k}$ of the corresponding creation operator $\hat{M}_{k}^{\dagger}$. It is easy to check that they satisfy

$$
\left[\hat{M}_{k}, \hat{M}_{k^{\prime}}\right]=\left[\hat{M}_{k}^{\dagger}, \hat{M}_{k^{\prime}}^{\dagger}\right]=0 \text {. }
$$

Furthermore, in condensed matter physics, it is often said that [9] magnons are approximately bosons when their density is low. This is "intuitively" denoted by

$$
\left[\hat{M}_{k}, \hat{M}_{k^{\prime}}^{\dagger}\right] \simeq \delta_{k, k^{\prime}}
$$

where the mathematically exact meaning of " $\simeq$ " depends on the context. Low-energy excited states of magnetic Hamiltonians are often approximated very well by fewmagnon states, such as $\hat{M}_{k_{1}}^{\dagger}|\psi\rangle$ and $\hat{M}_{k_{1}}^{\dagger} \hat{M}_{k_{2}}^{\dagger}|\psi\rangle$ with an appropriate vacuum state $|\psi\rangle$ [5]. Furthermore, a state where a macroscopic number of magnons are condensed is often used to describe the behavior of a macroscopic order when the many-body system exhibits a phase transition [9]. The condensation of magnons has been experimentally observed [18].

\section{BOSONIC CHARACTER AND ENTANGLEMENT}

In Refs. [13, 14], the quantity $\left\langle n\left|\left[\hat{c}, \hat{c}^{\dagger}\right]\right| n\right\rangle$ was used as an indicator of the bosonic character of composite fermions, where $\hat{c}^{\dagger}$ and $\hat{c}$ are creation and annihilation operators of the composite fermions, respectively, and $|n\rangle$ is the state on which $n$ composite fermions are excited. Here, we consider the similar quantity $\left|\left\langle\psi\left|\left[\hat{M}_{k}, \hat{M}_{k}^{\dagger}\right]\right| \psi\right\rangle\right|$ as the indicator of the bosonic character of magnons. This quantity should be 1 if magnons are exactly bosons. However, this quantity is upper bounded as [19]

$$
\begin{aligned}
\left|\left\langle\psi\left|\left[\hat{M}_{k}, \hat{M}_{k}^{\dagger}\right]\right| \psi\right\rangle\right| & =\frac{1}{N}\left|\sum_{l=1}^{N}\left\langle\psi\left|\left[\hat{\sigma}_{l}^{-}, \hat{\sigma}_{l}^{+}\right]\right| \psi\right\rangle\right| \\
& \leq \frac{1}{N} \sum_{l=1}^{N}\left|\left\langle\psi\left|\hat{\sigma}_{l}^{z}\right| \psi\right\rangle\right| \\
& \leq \frac{1}{N} \sum_{l=1}^{N} \sqrt{1-S_{l}} \equiv \Lambda,
\end{aligned}
$$

where $S_{l} \equiv 2\left[1-\operatorname{Tr}\left(\hat{\rho}_{l}^{2}\right)\right]$ is the entanglement between $l$ th spin and other spins [13, 14], $\hat{\rho}_{l} \equiv \operatorname{Tr}_{l}(|\psi\rangle\langle\psi|)$ is the reduced density operator for $l$ th spin, $\operatorname{Tr}_{l}$ means the trace over all spins except for $l$ th spin, and we have used the relation [20]

$$
\begin{aligned}
1-S_{l} & =\left\langle\psi\left|\hat{\sigma}_{l}^{x}\right| \psi\right\rangle^{2}+\left\langle\psi\left|\hat{\sigma}_{l}^{y}\right| \psi\right\rangle^{2}+\left\langle\psi\left|\hat{\sigma}_{l}^{z}\right| \psi\right\rangle^{2} \\
& \geq\left\langle\psi\left|\hat{\sigma}_{l}^{z}\right| \psi\right\rangle^{2}
\end{aligned}
$$

Here $\hat{\sigma}_{l}^{x}$ and $\hat{\sigma}_{l}^{y}$ are Pauli's $x$ and $y$ operators on site $l$, respectively. $S_{l}$ satisfies $0 \leq S_{l} \leq 1$. If $l$ th spin is maximally entangled with other spins, $S_{l}=1$. On the other hand, if $l$ th spin is separable from other spins, $S_{l}=0$. The physical meaning of Eq. (11) is that if the multipartite entanglement in $|\psi\rangle$ is strong in the sense that $S_{l}$ is close to 1 for many l's, then $\left|\left\langle\psi\left|\left[\hat{M}_{k}, \hat{M}_{k}^{\dagger}\right]\right| \psi\right\rangle\right| \ll 1$, which means magnons are not bosons.

\section{EXAMPLES}

Let us investigate consequences of Eq. (1) by studying some examples. First, we consider the Heisenberg ferromagnet:

$$
-\sum_{l=1}^{N}\left[\hat{\sigma}_{l}^{x} \hat{\sigma}_{l+1}^{x}+\hat{\sigma}_{l}^{y} \hat{\sigma}_{l+1}^{y}+\hat{\sigma}_{l}^{z} \hat{\sigma}_{l+1}^{z}\right] .
$$

A ground state of this Hamiltonian is $\bigotimes_{l=1}^{N}|0\rangle_{l}$ [21], and therefore we obtain $S_{l}=0$ for all $l$. This means 
$\Lambda=1$ and Eq. (1) does not prohibit magnons from being bosons. Indeed, the Heisenberg ferromagnet is a canonical example in the statistical physics where few magnons on the ground state behave as bosons [9, 22].

Second, let us consider the condensation of magnons. The state where $m$ magnons are condensed in wavenumber 0 is the $m$-Dicke state:

$$
\left(\sum_{l=1}^{N} \hat{\sigma}_{l}^{+}\right)^{m} \bigotimes_{l=1}^{N}|0\rangle_{l} .
$$

Let $m=\alpha N$, where $0<\alpha \leq 1 / 2$. It is easy to show that $S_{l}=4 \alpha(1-\alpha)$ for all $l$. Therefore, $\Lambda=1-2 \alpha<1$. (In particular, if $\alpha=1 / 2, \Lambda=0$.) This means that if a macroscopic number of magnons are condensed, they do not behave as bosons. Indeed, it is often pointed out that magnons cease to be bosons if their density is high [9] .

Third, let us consider the one-way quantum computer [15]. The one-way quantum computation is performed by adaptively measuring each qubit in the cluster state, which is defined as the simultaneous eigenvector of operators $\hat{K}_{l}(l=1,2, \ldots, N)$ :

$$
\hat{K}_{l} \equiv \hat{\sigma}_{l}^{x} \prod_{m \in C(l)} \hat{\sigma}_{m}^{z}
$$

corresponding to the simultaneous eigenvalue 1 , where $C(l)$ is the set of nearest-neighbor sites of $l$. To analyze the behavior of elementary excitations on the cluster state is important from the view point of the faulttolerant one-way quantum computation, since the propagation of an error is in the shape of elementary excitations. It is easy to understand that $S_{l}>0$ for all $l$ in the cluster state, since otherwise a single site is separable from other sites and therefore a measurement on this site does not contribute to the quantum computation. In fact, it can be shown by a direct calculation that $S_{l}=1$ for all $l[15]$. Therefore, we conclude that magnons on the cluster state are not bosons.

Fourth, let us consider the transverse Ising model:

$$
-\sum_{l=1}^{N}\left[\hat{\sigma}_{l}^{z} \hat{\sigma}_{l+1}^{z}+B \hat{\sigma}_{l}^{x}\right]
$$

where $B$ is the transverse magnetic field. This model exhibits a quantum phase transition at $B=1$ [17]. When $0<B \ll 1$, the ground state is approximately [23] the $N$-qubit Greenberger-Horne-Zeilinger (GHZ) state $\frac{1}{\sqrt{2}}\left(\left|0^{\otimes N}\right\rangle+\left|1^{\otimes N}\right\rangle\right)[27]$. Then, it is obvious that $S_{l}=1$ for all l's. Therefore, $\Lambda=0$ and this means magnons are not bosons. (Indeed, it is well-known that the transverse Ising model is well described by a set of fermions through the Jordan-Wigner transformation [17].) Note that even if we redefine the creation operator of a magnon by using another local operator $\hat{a}_{l}^{\dagger}$ as

$$
\frac{1}{\sqrt{N}} \sum_{l=1}^{N} e^{i k l} \hat{a}_{l}^{\dagger}
$$

"magnons" defined in this way are still not bosons, since the quantity $\left|\left\langle\psi\left|\left[\hat{a}_{l}, \hat{a}_{l}^{\dagger}\right]\right| \psi\right\rangle\right|$, which is the expectation value of a local operator on site $l$, is small if the mixedness of the reduced density operator $\hat{\rho}_{l}$ of site $l$ is strong. This is one explanation why a non-local transformation, such as the Jordan-Wigner transformation, is necessary for the transverse Ising model.

Fifth, we can also consider more complicated ground states. For example, in Ref. [6], the block entanglement in the ground state of the XY model was calculated. According to their result, $S_{l}>0$ for various parameters, and therefore Eq. (11) suggests that magnons are not bosons. Indeed, as is explained there, the XY model is exactly solved by introducing fermions through the Wigner-Jordan transformation.

Finally, the stability of quantum error-correcting codes is also important. For example, let us consider Kitaev's toric code [16]. It is easy to understand $S_{l}>0$ for all $l$, since otherwise a single site is separable from other sites and therefore an error on this site cannot be detected by syndrome measurements. In fact, it is well known that elementary excitations in the toric code are nonlocal anyons [16].

\section{CONCLUSION AND DISCUSSION}

In this Rapid Communication, we have studied how the multipartite entanglement in the vacuum state governs the bosonic character of magnons excited there. We have shown that if the multipartite entanglement is strong, magnons cease to be bosons.

As is well known, there are many different kinds of measures for multipartite entanglement, and each measure sees different aspects of a quantum many-body system. What we have considered here is an average of local entanglement. Such a way of quantifying multipartite entanglement is often adopted by many researchers. Among them, the most famous definition would be the "global entanglement"

$$
G \equiv 2\left[1-\frac{1}{N} \sum_{l=1}^{N} \operatorname{Tr}\left(\hat{\rho}_{l}^{2}\right)\right]
$$

given by Meyer and Wallach [28]. Here, let us point out that the plausible relation

$$
\left|\left\langle\psi\left|\left[\hat{M}_{k}, \hat{M}_{k}^{\dagger}\right]\right| \psi\right\rangle\right| \stackrel{?}{\leq} 1-G
$$

does not hold because of the counter example: $|\psi\rangle=$ $\sqrt{\frac{3}{4}}\left|0^{\otimes N}\right\rangle+\sqrt{\frac{1}{4}}\left|1^{\otimes N}\right\rangle$, which gives $\left|\left\langle\psi\left|\left[\hat{M}_{k}, \hat{M}_{k}^{\dagger}\right]\right| \psi\right\rangle\right|=$ $\frac{1}{2}>\frac{1}{4}=1-G$. It would be an interesting subject of future study to clarify which types of multipartite entanglement measures govern the bosonic character of magnons.

An important physical application of our result would be the quantum memory [29]. Quantum information encoded on a photon can be mapped to the collective excitation of atoms, and this information is later retrieved 
on demand. Since this collective excitation is in a shape of magnons, our result suggests that the "blank" state of the memory should not be highly entangled if one wants to preserve the bosonic character of magnons which carries the quantum information of incoming photons.

Finally, although we have considered a discrete field (spin lattice) here, it would be interesting to generalize our result to a continuous field, since there are several works studying the violation of Bell's inequality at the vacuum of a continuous field [30].

\section{Acknowledgments}

The author acknowledges the support by the French Agence Nationale de la Recherche (ANR) under the grant StatQuant (JC07 07205763).
[1] M. A. Nielsen and I. L. Chuang, Quantum Computation and Quantum Information (Cambridge University Press, Cambridge, UK, 2000).

[2] L. Amico, R. Fazio, A. Osterloh, and V. Vedral, Rev. Mod. Phys. 80, 517 (2008).

[3] O. Gühne and G. Toth, Phys. Rep. 474, 1 (2009).

[4] R. Horodecki, P. Horodecki, M. Horodecki, and K. Horodecki, Rev. Mod. Phys. 81, 865 (2009).

[5] G. D. Mahan, Many-particle physics (Kluwer Academic, New York, 2000).

[6] J. I. Latorre, E. Rico, and G. Vidal, Quant. Inf. Comput. 4, 48 (2004).

[7] A. Osterloh, L. Amico, G. Falci, and R. Fazio, Nature 416, 608 (2002).

[8] F. Verstraete and J. I. Cirac, Phys. Rev. B 73, 094423 (2006).

[9] S. Nakajima, Y. Toyozawa, and R. Abe, The physics of elementary excitations (Springer Berlin, Berlin, 1980).

[10] T. Morimae, A. Sugita, and A. Shimizu, Phys. Rev. A 71, 032317 (2005).

[11] M. Blasone, F. Dell'Anno, S. De Siena, and F. Illuminati, Phys. Rev. A 77, 062304 (2008).

[12] S. Bose, Comtemp. Phys. 48, 13 (2007).

[13] C. K. Law, Phys. Rev. A 71, 034306 (2005).

[14] C. Chudzicki, O. Oke, and W. K. Wootters, Phys. Rev. Lett. 104, 070402 (2010).

[15] R. Raussendorf and H. J. Briegel, Phys. Rev. Lett. 86, 5188 (2001).

[16] A. Yu. Kitaev, Ann. Phys. 303, 2 (2003).

[17] S. Sachdev, Quantum Phase Transition, (Cambridge University Press, Cambridge, UK, 1999).

[18] E. Della Torre, L. H. Bennett, and R. E. Watson, Phys. Rev. Lett. 94, 147210 (2005); T. Radu, H. Wilhelm, V. Yushankhai, D. Kovrizhin, R. Coldea, Z. Tylczynski, T. Lühmann, and F. Steglich, Phys. Rev. Lett. 95, 127202 (2005); T. Nikuni, M. Oshikawa, A. Oosawa, and H. Tanaka, Phys. Rev. Lett. 84, 5868 (2000).

[19] We obtain the same inequality even if we consider $\left[\hat{M}_{k}, \hat{M}_{k^{\prime}}^{\dagger}\right]$ since $\left|e^{-i k l+i k^{\prime} l^{\prime}}\right|=1$.

[20] The first equality is shown by seeing that a state $\hat{\rho}_{l}$ is generally written as $\hat{\rho}_{l}=\frac{1}{2}\left(\operatorname{Tr}\left(\hat{\rho}_{l} \hat{\sigma}_{l}^{x}\right) \hat{\sigma}_{l}^{x}+\operatorname{Tr}\left(\hat{\rho}_{l} \hat{\sigma}_{l}^{y}\right) \hat{\sigma}_{l}^{y}+\right.$ $\left.\operatorname{Tr}\left(\hat{\rho}_{l} \hat{\sigma}_{l}^{z}\right) \hat{\sigma}_{l}^{z}+\hat{1}\right)$ and therefore the purity is given by $\operatorname{Tr}\left(\hat{\rho}_{l}^{2}\right)=\frac{1}{2}\left(\operatorname{Tr}\left(\hat{\rho}_{l} \hat{\sigma}_{l}^{x}\right)^{2}+\operatorname{Tr}\left(\hat{\rho}_{l} \hat{\sigma}_{l}^{y}\right)^{2}+\operatorname{Tr}\left(\hat{\rho}_{l} \hat{\sigma}_{l}^{z}\right)^{2}+1\right)$.

[21] Because of the rotational symmetry of the Hamiltonian, other product states where all spins point the same direction are also ground states. When another ground state than $\left|0^{\otimes N}\right\rangle$ is considered, the definition of the creation operator of a magnon should be appropriately modified.

[22] Indeed, it is easy to confirm that $\hat{M}_{k}^{\dagger} \bigotimes_{l=1}^{N}|0\rangle_{l}$ is the exact energy eigenstate of the Heisenberg ferromagnet, and that $\left(\bigotimes_{l=1}^{N}\left\langle\left. 0\right|_{l}\right)\left[\hat{M}_{k}, \hat{M}_{k^{\prime}}^{\dagger}\right]\left(\bigotimes_{l=1}^{N}|0\rangle_{l}\right)=\delta_{k, k^{\prime}}\right.$.

[23] In fact, the approximation of the ground state by the GHZ state is not a good one, since according to the Peron-Frobenius theorem [24], the ground state of the transverse Ising model has no node, that is, the ground state is a superposition of all $2^{N} N$-qubit computational basis. However, since the contribution of $\bigotimes_{l=1}^{N}|0\rangle_{l}$ and $\bigotimes_{l=1}^{N}|1\rangle_{l}$ to the superposition is large compared with that by other computational basis [25], such approximation is convenient to capture the essence. In Ref. [25], it was shown that the exact ground state of the transverse Ising model for $0<B<1$ has a superposition of macroscopically distinct states. According to the result of Ref. [26], such macroscopic superposition means a strong multipartite entanglement in the sense that $S_{l}=O(1)$ for a macroscopic number $[O(N)]$ of $l$ 's, where $f(N)=O\left(N^{r}\right)$ means $\lim _{N \rightarrow \infty} f(N) / N^{r}=$ const. $\neq 0$. If we consider the periodic boundary condition, this means $S_{l}=O(1)$ for all $l$ because of the translational symmetry. Then, $\Lambda<1$ and therefore we obtain the same conclusion that magnons are not bosons.

[24] R. A. Horn and C. R. Johnson, Matrix Analysis (Cambridge University Press, Cambridge, UK, 1990).

[25] T. Morimae, Phys. Rev. A 81, 022304 (2010).

[26] T. Morimae, Phys. Rev. A 81, 010101(R) (2010).

[27] If a symmetry breaking field is introduced, the symmetry of the ground state is broken, and one of two separable states $\left|0^{\otimes N}\right\rangle$ or $\left|1^{\otimes N}\right\rangle$ is obtained. In this case, we obtain the bosonic magnon since these symmetry-broken ground states are separable.

[28] D. A. Meyer and N. R. Wallach, J. Math. Phys. 43, 4273 (2002).

[29] A. I. Lvovsky, B. C. Sanders, and W. Tittel, Nature Photonics, 3, 706 (2009).

[30] S. J. Summers and R. F. Werner, Phys. Lett. A 110, 257 (1985). 